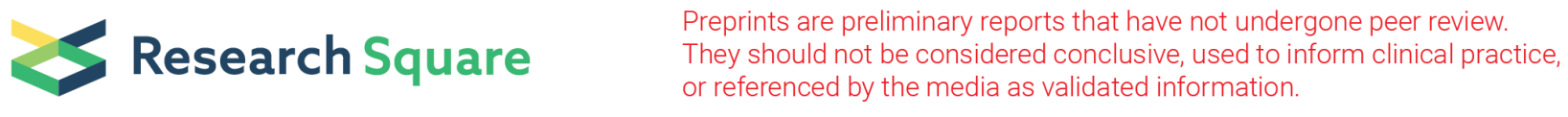

\title{
Multidimensional Fatigue in Chinese Meningioma Patients Newly Diagnosed: Prevalence, Severity and Risk Factors
}

Dandan Zhang

Affiliated Hospital of Nantong University

Qian Wu

Affiliated Hospital of Nantong University

Xixi Gu

Affiliated Hospital of Nantong University

Zhifeng Gu

Affiliated Hospital of Nantong University

Chen Dong

Affiliated Hospital of Nantong University

Rong Li

Affiliated Hospital of Nantong University

Zhiying Zong

Affiliated Hospital of Nantong University

Xiaomei Zhang ( $\nabla$ tdfyzxm@163.com)

Affiliated Hospital of Nantong University https://orcid.org/0000-0002-1477-9145

Liren Li

Affiliated Hospital of Nantong University

Research Article

Keywords: Meningioma, Fatigue, Quality of life, Multidimensional assessment

Posted Date: June 11th, 2021

DOl: https://doi.org/10.21203/rs.3.rs-591942/v1

License: @ (1) This work is licensed under a Creative Commons Attribution 4.0 International License. Read Full License 


\section{Abstract \\ Purpose}

Few studies have assessed fatigue of meningioma patients. The purpose of this study was to explore the prevalence, severity, and risk factors of multidimensional fatigue, as well as the impact on health-related quality of life (HRQoL) in Chinese meningioma patients newly diagnosed.

\section{Methods}

This cross-sectional study included 120 Chinese meningioma patients recruited from Affiliated Hospital of Nantong University from January 2020 to February 2021. Data were collected before surgery, including demographic, clinical and psychological characteristics, as well as fatigue scores, based on completion of the Multidimensional Fatigue Inventory (MFI-20). Spearman correlation and multiple linear regression were used to analyze the data.

\section{Results}

The results demonstrated that a high prevalence of severe fatigue for each dimension: general fatigue (33.3\%), physical fatigue (27.5\%), reduced activity (28.3\%), reduced motivation (12.5\%), mental fatigue (11.7\%), and total fatigue (23.3\%). Headache and anxiety were predictors of general fatigue. Depression was an independent predictor of physical fatigue. Karnofsky Performance Status (KPS) score and depression independently predicted reduced activity. Depression and Epworth Sleepiness Scale (ESS) score were risk factors predicting reduced motivation, while KPS score and anxiety predicted mental fatigue. Importantly, comorbidity, KPS score, headache, depression, sleep disturbances, and ESS score could predict total fatigue. Furthermore, MFI-20 scores in all dimensions were negatively correlated with Short Form 36 Health Survey (SF-36) scores in all dimensions.

\section{Conclusion}

Our findings indicate that meningioma patients newly diagnosed are frequently affected by fatigue, potentially contributing to impair HRQoL. For patients with risk factors of fatigue, targeted interventions are advised in order to decrease fatigue and improve HRQoL.

\section{Introduction}

Meningiomas arising from the arachnoid cap cells are the most common primary central nervous tumor, accounting for more than one third of all intracranial tumors, with rising incidence in adults aged 65 years and older[1]. The female-to-male ratio is 2:1, and more than $90 \%$ of meningiomas are classified as benign $[2,3]$. Owing to mass effect and peritumoral edema, meningioma patients may suffer from a significant symptom burden, often from the time of diagnosis[4]. Symptoms of meningioma can be specific, such as epilepsy, neurological and neurocognitive deficits, visual impairment, and anosmia[1,5]. In addition, the majority symptoms are more general, such as headache, anxiety, depression, sleep disturbance and fatigue[6]. These symptoms may cause limitations of patients' personal and professional activities, eventually leading to the deterioration of patients' health-related quality of life (HRQoL).

Fatigue, an important part of patients' initiative reporting of prognosis and quality of life, is one of the most common and distressing symptoms in patients with primary brain tumors throughout the disease trajectory, with a prevalence of 34\%-96\%[7-9]. Fatigue, characterized as a subjective experience of persistent and extreme exhaustion, a lack of energy, and tiredness, is a multidimensional concept including physiological, psychological, and social aspects[10,11]. It has a negative impact on work, social relationships, mood, and daily activities, is a strong predictor of decreased patients' satisfaction and HRQoL[12]. To improve patients' HRQoL, it is extremely important to explore the severity of fatigue and evaluated associated risk factors. Some factors recognized to contribute to fatigue in solid tumor population, are pain, emotional distress, sleep disturbances, low activity levels, malnutrition, medication side effects, and comorbidities, as well as tumor-related factors and complications[13]. Nevertheless, few studies have addressed the problem of fatigue in brain tumor patients in depth, especially in meningioma patients.

Additionally, among these limited studies, there are several limitations have to be mentioned. Firstly, some studies included a broad range of brain tumors types, not just meningiomas[14,15]. Secondly, fatigue was mainly evaluated as a oncological treatment complication, thereby failing to acknowledge the primary impact of the tumor itself and other treatment-independent factors[16]. Thirdly, some studies identified and quantified fatigue in a very rudimental way, using a single-item format or as part of a general symptom inventory, and did not investigate other manifestations of fatigue[17,18]. Finally, the incidence and risk factors for multidimensional fatigue in meningioma patients have not been clearly defined.

To address these issues, we conducted the first study, to our knowledge, of fatigue in Chinese meningioma patients newly diagnosed and ready for surgery using a multidimensional conceptualization. The aims of our study are therefore (1) to explore meningioma patients' prevalence and severity of fatigue, (2) to determine contributing factors of fatigue in meningioma patients, and (3) to investigate the relationship between the fatigue dimensions and HRQoL.

\section{Methods}

\section{Participants}

This was a cross-sectional study that was conducted in the Department of Neurosurgery in Affiliated Hospital of Nantong University from January 2020 to February 2021. All eligible patients were histologically confirmed as intracranial meningioma by a neuropathologist according to the 2016 World Health Organization-classification[19] and completed self-report questionnaires on the day before surgery. Subjects who met any of the following criteria were 
excluded: (1) they were less than 18 years old, (2) a severe hepatic, hematological, cardiovascular or renal disease that caused chronic fatigue and impaired quality of life,(3) patients with a history of severe psychiatric or neurological disorders, and cannot cooperate with the completion of the questionnaire. This study was approved by the Ethics Committee of the Affiliated Hospital of Nantong University (number:2020-K042). All participants completed the questionnaire on a voluntary basis and written informed consent were obtained from all subjects, according to the Declaration of Helsinki.

\section{Demographic and clinical characteristics}

Demographic variables were self-reported by patients during a standardized interview. Medical history and laboratory data were retrieved from the electronic medical records for each patient at the time of the visit. Demographic variables include gender, age, body mass index (BMI), place of residence, marital status, educational level, employment, yearly per capita income, medical insurance, tobacco use, alcohol use, physical exercise and menopausal status. Clinical variables include time since diagnosis, comorbidity, hospitalization days, initial presenting symptoms, tumor size (which was measured as the maximum diameter), numbers, lateralization, location and WHO grade. Routine laboratory measures included hemoglobin ( $\mathrm{g} / \mathrm{L}$ ), erythrocyte count ( $\mathrm{g} / \mathrm{L}$ ), serum albumin $(\mathrm{g} / \mathrm{L})$, serum potassium $(\mathrm{mmol} / \mathrm{L})$ and serum sodium $(\mathrm{mmol} / \mathrm{L})$.

\section{Assessment of patient-reported outcomes}

Functional status was measured with the Barthel Index (BI)[20] and Karnofsky Performance Status (KPS) scale[21]. The ten-item BI evaluates impairment in activities of daily living, and total BI score ranges from 0 to 100, with higher scores indicating greater functional independence. The KPS is a scale indicating a person's overall functional outcome (person's ability to work, physical activity, and self-care). The KPS index is assessed by the health care provider on a scale of 0 to 100 , with 100 being functionally healthy and having no symptoms of disease.

Cognitive functioning was evaluated using the Mini Mental State Examination (MMSE)[22] that is widely used instrument for assessment of subjective cognitive functioning in clinical settings and for research purposes. MMSE scores range between 0 and 30 , with higher scores indicating better cognitive functioning.

The severity of depression and anxiety symptoms was evaluated with the Hospital Anxiety and Depression Scale (HADS) that is comprised of two seven-item subscales of Depression (HADS-D) and Anxiety (HADS-A). Each item has a 4-point Likert scale and is scored between 0 and 3 . Total score on each subscale ranges from 0 to 21 with greater score indicating greater symptom severity. Patients scoring 8 or above in any of the scales are classified as clinically relevant anxiety and depression respectively. The HADS is the most frequently applied measure in brain tumor patients with good psychometric properties[23,24].

Sleep quality was measured using the Pittsburgh Sleep Quality Index (PSQI)[25], an established international measure of sleep quality. The PSQI consists of 19 questions grouped in seven components, relates to the last 1-month time interval. Each answer provides a score ranges from 0 to 3 , that are summed in a global PSQI score that ranges from 0 to 21 . Higher scores indicate worse subjective sleep quality. Sleep disturbances are defined as a PSQI score of > 5 .

Daytime sleepiness was quantified using the Epworth Sleepiness Scale (ESS), which consists of eight self-rated items with scores from 0 to 3 , that measure a subject's habitual "possibility of dozing or falling sleep" in common situations of daily life. The total ESS score ranges from 0 to 24 , with higher scores reflecting greater sleepiness[26].

Fatigue was assessed using the Multidimensional Fatigue Inventory (MFI-20), it is a widely used multidimensional questionnaire with adequate psychometric quality[27]. The MFI-20 has been developed specifically to measure cancer-related fatigue[28] and has been recommended for use with brain tumor patients[29]. It contains a total of 20 items, including five domain scores (general fatigue, physical fatigue, reduced activity, reduced motivation, and mental fatigue). Each domain has 4 items and is scored on a 5-point Likert type scale to give domains scores of 4-20. A higher score indicates more fatigue. Severe fatigue is considered present for a dimension if the dimension score is $>12[30]$. The total score is the sum of the five-dimensional fatigue scores, and ranges from 20 to 100, where > 60 indicates severe total fatigue[31].

We used the Short Form 36 Health Survey (SF-36) questionnaire to evaluate HRQoL. It is an internationally recognized universal scale for evaluating quality of life in neuro-oncology setting within the previous 4 weeks[32]. It mainly includes 36 statements, with 8 dimensions: physical functioning (PF), role physical $(\mathrm{RP})$, body pain (BP), general health (GH), vitality (VT), social functioning (SF), role emotional problems (RE), and mental health (MH). Raw scores are converted linearly to 0-100 scale scores, with higher scores indicating better HRQoL. In addition, higher order component scores are calculated on two scales: a physical component scale (PCS) and a mental component scale (MCS)[33].

\section{Statistical analysis}

Descriptive statistics were calculated for all variables. Continuously variable data with normal distribution were presented as mean \pm standard deviation (SD), while descriptive statistics of skewed distribution often presented as median with interquartile ranges. Nominal data were presented as proportion (percentage). If the two continuous variables accorded with normal distribution, we followed Pearson correlation to analyze data. If this condition was not met, Spearman correlation was used. All significative variables $(P<0.05)$ studied were entered into Multiple linear regression to identify potential predictors of fatigue in meningioma patients. The level of significance was set at $P$ value $<0.05$ (two-sided). All statistical analyses were performed using SPSS version 26.0.

\section{Results}

\section{Patient characteristics}


Of the 125 patients approached, 5 patients were excluded because they did not fully complete the provided questionnaires. Eventually, 120 meningioma patients fully participated in this study. Results indicated that the mean (SD) age of meningioma patients was $59.29 \pm 11.38$ years, $73.3 \%$ of them were females. The median time since diagnosis of meningioma patients was 0.33 months, and $55.8 \%$ patients had comorbidities. The majority of patients initially presented with intracranial hypertension (63.3\%), and $23.8 \%$ of the patients complained of headache during the disease course. The WHO grade at the time of resection was used to assign the WHO grade. The meningioma was WHO grade I for $73.5 \%$ of the patients, grade II for $23.9 \%$, and anaplastic meningioma WHO grade III for $2.6 \%$ of the patients. The median tumor size was $3.6 \mathrm{~cm}$. Roughly a third of the patients in our study had meningiomas located in the skull base. The median $\mathrm{BI}$ and KPS scores of the meningioma patients were 100 and 90, respectively. Meningioma patients' demographic, clinical, and psychological characteristics are further summarized in Table 1 and Table 2.

\section{Prevalence and severity of fatigue in meningioma patients}

The MFI-20 five subscales and total scores are shown in Table 3. By using the MFI-20 questionnaire, the median (interquartile range) scores of multidimensional fatigue were as follows: general fatigue, $10(8,13.75)$, physical fatigue, $6(4,13)$, reduced activity, $9(8,14)$, reduced motivation, $8(7,10)$, mental fatigue, $8(8,10)$, and total fatigue, $43(36,57.75)$, respectively. Across all the subscales of MFI-20 questionnaire, severe fatigue level was reported by up to $33.3 \%$ in general fatigue subscale, followed by reduced activity subscale (28.3\%), physical fatigue subscale (27.5\%), reduced motivation subscale (12.5\%) and mental fatigue subscale (11.7\%). In addition, 28 patients had total fatigue scores $>60$, and the prevalence of severe total fatigue was $23.3 \%$.

\section{Correlations between demographic, clinical, and psychological characteristics and fatigue in meningioma patients}

Spearman rank correlation coefficients were computed to identify the relationships between demographic, clinical, and psychological characteristics and fatigue in meningioma patients. As shown in Table 1 , patients with unemployment $(P=0.047,0.045,0.046,0.010)$, lower yearly per capita income $(P=0.001$, $0.002,<0.001,<0.001)$, and postmenopause $(P=0.003,0.025,0.025,0.001)$ were more likely to have higher fatigue scores in total fatigue, physical fatigue score, reduced activity and reduced motivation. In addition, we also found women with meningioma had higher fatigue score in general fatigue $(P=0.005)$. Besides, age was found to be positively associated with reduced activity scores $(P=0.013)$ and reduced motivation scores $(P<0.001)$.

As indicated in Table 2, we found patients with comorbidity $(P=0.042,0.049)$ and headache $(P=0.034,0.005)$ had more total fatigue and more general fatigue. Tumor size had a positive association with total fatigue score $(P=0.002)$, physical fatigue score $(P=0.001)$, reduced activity score $(P=0.004)$ and reduced motivation score $(P=0.002)$. Higher histological grade was correlated with more total fatigue $(P=0.032)$ and more physical fatigue $(P=0.034)$. Moreover, higher serum sodium was associated with increases in total fatigue $(P=0.025)$, reduced activity $(P=0.033)$ and reduced motivation $(P<0.001)$. Additionally, MMSE scores had negatively associations with total fatigue and four dimensions of MFI-20 except mental fatigue. ESS scores were positively related to total fatigue and four dimensions of MFI-20 except general fatigue. BI and KPS scores were negatively correlated with total fatigue and five dimensions of MFI-20. Patients with anxiety or depression or sleep disturbance suffered from more fatigue (total fatigue and all dimensions).

\section{Risk factors for fatigue in meningioma patients}

Multivariate linear regression was used to explore possible predictors of multidimensional fatigue of meningioma patients newly diagnosed by assessing all of the associated variables described above, including demographic, clinical and psychological characteristics, as shown in Table 4. Headache and anxiety were risk factors predicting general fatigue, while depression predicted physical fatigue, reduced activity and reduced motivation. Furthermore, KPS score was a significant predictor for reduced activity and mental fatigue, ESS score was a predictor of reduced motivation, anxiety was a risk factor predicting mental fatigue, and comorbidity, KPS score, headache, depression, sleep disturbance, and ESS score were independent predictors of total fatigue in meningioma patients.

\section{Correlations between fatigue and quality of life in meningioma patients}

Table 5 presents the correlations between MFI-20 scores and SF-36 scores. MFI-20 scores in all dimensions were negatively correlated with SF-36 in all dimensions.

\section{Discussion}

This is the first cross-sectional study to explore predictors of fatigue and their impact on quality of life in patients with newly diagnosed meningiomas before surgery in China. Our findings indicate that fatigue is a prominent pretreatment symptom in meningioma patients, and the prevalence of severe total fatigue is $23.3 \%$, based on a MFI-20 total score $>60$. Furthermore, fatigue among meningioma patients is significantly associated with clinical characteristics (comorbidity, KPS score, headache), psychological problems (anxiety and depression), and sleep quality (sleep disturbance and ESS score).

Studies on fatigue in patients with brain tumors were more focused on gliomas with more aggressive and worse clinical prognosis[8,34-36]. However, as in gliomas, fatigue was frequently reported as a common and troublesome symptom before and after treatment for meningioma patients[9,18,37]. In our study, we conducted a more in-depth research of multidimensional fatigue in patients with newly diagnosed meningiomas. Indeed, among patients with newly diagnosed meningiomas, the prevalence of severe fatigue varied between $11.7 \%$ for mental fatigue and $33.3 \%$ for general fatigue. In a prior study by van der Linden et al with preoperative data that could be compared with ours the fatigue rate of each dimension was higher than that of our corresponding dimensions[9]. Differences in study design, patient selection, evaluation time point, and definition of severe fatigue may explain the differences in prevalence and hinder meaningful comparison between studies.

Only a limited number of studies have focused on the relationship between comorbidity and fatigue in brain tumor patients. In a prior study, the univariate analysis showed that comorbidity was significantly associated with fatigue in patients with brain tumors undergoing proton beam therapy, but the 
multivariate analysis did not further prove this relationship[38]. In this study, we found a significant association between comorbidity and total fatigue in meningioma patients. The majority of participants (age > 60 years) have other comorbid conditions that may affect the symptom of fatigue, such as cardiovascular disease[39] and diabetes[40]. It is unclear how comorbidities influence fatigue as these coexisting conditions may share a common pathophysiological pathway or share symptoms resulting in a synergistic symptom experience. Further studies with larger samples are needed to examine the summative and potentiating effects of comorbid conditions on fatigue symptom. Furthermore, our study did not examine which comorbid condition contributed to fatigue. Therefore, further research is needed to examine the contribution of specific comorbid conditions on fatigue.

Poor functional status is a well-known negative factor affecting the quality of life of meningioma patients[41]. Our study shows high correlations between KPS score and fatigue, and low KPS score is an independent factor for total fatigue, reduced activity, and mental fatigue. In accordance with our findings, a previous prospective study of fatigue in glioma patients indicated a significant relationship between low KPS and high fatigue before surgery, which also existed after surgery[35], while another study on gliomas found no relationship between KPS and fatigue after surgery[8]. It is necessary to explore in depth fatigue in meningioma patients at various stages in the disease trajectory.

Headache was another independent factor for total fatigue and general fatigue. Headache is the most frequent symptom and occurs in about two thirds of meningioma patients[42]. The headache of meningiomas may depend on compression of specific structures or an increase in intracranial pressure. Compared with other headaches, those associated with increased intracranial pressure are more likely to be severe, continuous, associated with nausea and vomiting, and refractory to analgesics. Patients may experience more negative emotions (e.g., anxiety, depression, fatigue) due to frequent and intense headache. A cross-sectional study conducted by Spierings et al. has shown higher levels of fatigue in patients with chronic headache[43]. Conversely, another crosssectional study found that fatigue was a risk factor for headache[44]. Hence, the nature of the causal relationship between fatigue and headache is unclear and longitudinal data involving a larger sample are required.

The results of the current study also revealed that anxiety and depression were significantly associated with fatigue in meningioma patients. Similarly, other researchers have established an association between fatigue, depression, and anxiety in studies of other primary brain tumors[8,45]. Moreover, one study argued that meningioma, over other types of tumors, can lead to greater levels of anxiety and depression, resulting in the aggravation of health-related complications[46]. Therefore, we recommend the routine screening of patients for psychological disorders in order to determine targeted interventions to help meningioma patients to get rid of fatigue.

Moreover, we also found that sleep quality was strongly correlated with fatigue, consistent with previous studies on primary brain tumors[14,15]. Sleep-wake disturbances, which include both alterations in sleep (insomnia) and daytime sleepiness, are frequently reported as the most severely rated symptoms within health-related quality of life across the disease course or treatments, along with fatigue, and in turn can contribute to other symptoms and psychopathology[47,48]. In our analysis, reduced motivation was more likely to occur in patients with more severe daytime sleepiness. In a previous study, primary brain-tumor patients with sleepiness also had lower quality of life, poor performance status and shorter survival[49]. In addition, although fatigue and sleepiness are considered as two distinct symptoms, they also show a great overlap and presumably, at least to some extent, similar pathophysiology[50]. Thus, sleepiness is undoubtedly associated with fatigue, which is confirmed by our findings. Clinically, our results suggest that interventions to improve sleep may be effective for meningioma patients with reduced motivation. Unfortunately, no study has examined an intervention specifically aimed at improving the sleep of brain tumor patients[48]. The interventions to alleviate fatigue and sleepiness need further research in the further.

Considering the importance of HRQoL in clinical neuro-oncology, our study also explored the impact of fatigue on HRQoL of meningioma patients. A largest prospective, longitudinal cross-sectional cohort study of $\mathrm{HRQOL}$ in postoperative meningiomas to date, indicated that fatigue was significantly associated with HRQoL[18]. In our analysis, we found a significant relation between all dimensions of fatigue (expressed by MFI-20) and all dimensions of HRQoL (expressed by SF-36) in meningioma patients newly diagnosed. It follows then that eliminating fatigue is very important to improve HRQoL. In clinical settings, early identification of patients at high risk for fatigue facilitates timely provision of information and intervention. At present, although only a few intervention studies have been conducted on fatigue in patients with brain tumors, we can take lessons from the cancer literature to apply to meningiomas. Psychotherapy such as cognitive-behavioral therapy to alter the way people behave has been shown to be successful in cope with fatigue and functional impairment in cancer survivors[51]. Psychological educational and lifestyle management interventions, such as energy conservation and activity management (ECAM), have been studied in multiple randomized controlled trials to demonstrate a positive impact on fatigue outcomes[52]. Support complementary therapies (e.g., qigong, yoga, hypnosis, and music therapy) may also offer some benefits to cancer patients[12]. Exercise and physical activity have demonstrated to improve cancer-related fatigue and overall quality of life[53]. However, for meningioma patients, these interventions and their effectiveness need to be further investigated.

To our knowledge, this is the first study to explore the multidimensional fatigue and its effects on HRQoL of meningioma patients newly diagnosed and ready for surgery in China. The fatigue assessment with the MFI-20 is beneficial because it includes multiple fatigue subtypes, allowing for stratification of these subtypes and identification of key issues. However, acknowledged limitations of this study should be considered that participants were recruited from a single neurosurgery clinic and sample size needed to be expanded. And, because of the cross-sectional in design, the current study only demonstrates associations but not causation. Therefore, further systematic studies from multiple centers and neurophysiological researches should be conducted to clarify effective interventions to reduce or treat fatigue, potentially resulting in improvement of HRQoL in meningioma patients.

\section{Conclusion}

This study presents valuable information on the aspects of fatigue and quality of life in meningioma patients newly diagnosed, revealing that fatigue is common and serious symptom in these patients, with predictors differing for each fatigue dimension. In addition, severe fatigue significantly reduces HRQoL 
in meningioma patients physically and psychologically. The current research is a necessary first step in investigating fatigue in meningioma patients, and assessment and treatment of fatigue need to be further investigated, potentially resulting in improvement of HRQoL.

\section{Declarations}

Funding This study was supported by Science and technology Project of Nantong City (Grant no. MS12020011).

Conflicts of interest/ Competing interests The authors declare that they have no conflict of interest.

Availability of data and material The datasets generated during and/or analysed during the current study are not publicly available due to privacy concerns but are available from the corresponding author on reasonable request.

Code availability Not applicable

Ethics approval This study was performed in line with the principles of the Declaration of Helsinki. Approval was granted by the Ethics Committee of the Affiliated Hospital of Nantong University (No. 2020-K042).

Consent to participate Informed consent was obtained from all individual participants included in the study.

Consent for publication Not applicable

Acknowledgements The authors are thankful to Zhifeng Gu, Chen Dong, Rong Li, and Zhiying Zong for their assistance with this paper as well as all patients who participated in the study.

\section{References}

1. Fathi AR, Roelcke U (2013) Meningioma. Curr Neurol Neurosci Rep 13(4):337. doi:10.1007/s11910-013-0337-4

2. Riemenschneider MJ, Perry A, Reifenberger G (2006) Histological classification and molecular genetics of meningiomas. Lancet Neurol 5(12):1045-1054. doi:10.1016/S1474-4422(06)70625-1

3. Wiemels J, Wrensch M, Claus EB (2010) Epidemiology and etiology of meningioma. J Neurooncol 99(3):307-314. doi:10.1007/s11060-010-0386-3

4. Armstrong TS, Cohen MZ, Eriksen LR, Hickey JV (2004) Symptom clusters in oncology patients and implications for symptom research in people with primary brain tumors. J Nurs Scholarsh 36(3):197-206. doi:10.1111/j.1547-5069.2004.04038.x

5. Buerki RA, Horbinski CM, Kruser T, Horowitz PM, James CD, Lukas RV (2018) An overview of meningiomas. Future Oncol 14(21):2161-2177. doi:10.2217/fon-2018-0006

6. Zamanipoor Najafabadi AH, Peeters MCM, Dirven L, Lobatto DJ, Groen JL, Broekman MLD, Peerdeman SM, Peul WC, Taphoorn MJB, van Furth WR (2017) Impaired health-related quality of life in meningioma patients-a systematic review. Neuro Oncol 19(7):897-907. doi:10.1093/neuonc/now250

7. Fox SW, Lyon D, Farace E (2007) Symptom clusters in patients with high-grade glioma. J Nurs Scholarsh 39(1):61-67. doi:10.1111/j.15475069.2007.00144.x

8. Valko PO, Siddique A, Linsenmeier C, Zaugg K, Held U, Hofer S (2015) Prevalence and predictors of fatigue in glioblastoma: a prospective study. Neuro Oncol 17(2):274-281. doi:10.1093/neuonc/nou127

9. van der Linden SD, Gehring K, Rutten GM, Kop WJ, Sitskoorn MM (2020) Prevalence and correlates of fatigue in patients with meningioma before and after surgery. Neurooncol Pract 7(1):77-85. doi:10.1093/nop/npz023

10. Aaronson LS, Teel CS, Cassmeyer V, Neuberger GB, Pallikkathayil L, Pierce J, Press AN, Williams PD, Wingate A (1999) Defining and measuring fatigue. Image J Nurs Sch 31(1):45-50. doi:10.1111/j.1547-5069.1999.tb00420.x

11. Trendall J (2000) Concept analysis: chronic fatigue. J Adv Nurs 32(5):1126-1131. doi:10.1046/j.1365-2648.2000.01583.x

12. Bower JE, Bak K, Berger A, Breitbart W, Escalante CP, Ganz PA, Schnipper HH, Lacchetti C, Ligibel JA, Lyman GH, Ogaily MS, Pirl WF, Jacobsen PB, American Society of Clinical O (2014) Screening, assessment, and management of fatigue in adult survivors of cancer: an American Society of Clinical oncology clinical practice guideline adaptation. J Clin Oncol 32(17):1840-1850. doi:10.1200/JC0.2013.53.4495

13. Berger AM, Mooney K, Alvarez-Perez A, Breitbart WS, Carpenter KM, Cella D, Cleeland C, Dotan E, Eisenberger MA, Escalante CP, Jacobsen PB, Jankowski C, LeBlanc T, Ligibel JA, Loggers ET, Mandrell B, Murphy BA, Palesh O, Pirl WF, Plaxe SC, Riba MB, Rugo HS, Salvador C, Wagner LI, Wagner-Johnston ND, Zachariah FJ, Bergman MA, Smith C, National comprehensive cancer n (2015) Cancer-Related Fatigue, Version 2.2015. J Natl Compr Canc Netw 13(8):1012-1039. doi:10.6004/jnccn.2015.0122

14. Kim BR, Chun MH, Han EY, Kim DK (2012) Fatigue assessment and rehabilitation outcomes in patients with brain tumors. Support Care Cancer 20(4):805-812. doi:10.1007/s00520-011-1153-5

15. Armstrong TS, Cron SG, Bolanos EV, Gilbert MR, Kang DH (2010) Risk factors for fatigue severity in primary brain tumor patients. Cancer 116(11):27072715. doi:10.1002/cncr.25018

16. Ozbayir T, Malak AT, Bektas M, Ilce AO, Celik GO (2011) Information needs of patients with meningiomas. Asian Pac J Cancer Prev 12(2):439-441

17. Kalasauskas D, Keric N, Abu Ajaj S, von Cube L, Ringel F, Renovanz M (2020) Psychological Burden in Meningioma Patients under a Wait-and-Watch Strategy and after Complete Resection Is High-Results of a Prospective Single Center Study. Cancers (Basel) 12 (12). doi:10.3390/cancers12123503 
18. Nassiri F, Price B, Shehab A, Au K, Cusimano MD, Jenkinson MD, Jungk C, Mansouri A, Santarius T, Suppiah S, Teng KX, Toor GS, Zadeh G, Walbert T, Drummond KJ, International Consortium on M (2019) Life after surgical resection of a meningioma: a prospective cross-sectional study evaluating health-related quality of life. Neuro Oncol 21(Suppl 1):i32-i43. doi:10.1093/neuonc/noy152

19. Louis DN, Perry A, Reifenberger G, von Deimling A, Figarella-Branger D, Cavenee WK, Ohgaki H, Wiestler OD, Kleihues P, Ellison DW (2016) The 2016 World Health Organization Classification of Tumors of the Central Nervous System: a summary. Acta Neuropathol 131(6):803-820. doi:10.1007/s00401-0161545-1

20. Mahoney FI, Barthel DW (1965) Functional Evaluation: The Barthel Index. Md State Med J 14:61-65

21. Schag CC, Heinrich RL, Ganz PA (1984) Karnofsky performance status revisited: reliability, validity, and guidelines. J Clin Oncol 2(3):187-193. doi:10.1200/JC0.1984.2.3.187

22. Folstein MF, Folstein SE, McHugh PR (1975) "Mini-mental state". A practical method for grading the cognitive state of patients for the clinician. J Psychiatr Res 12(3):189-198. doi:10.1016/0022-3956(75)90026-6

23. Rooney AG, Carson A, Grant R (2011) Depression in cerebral glioma patients: a systematic review of observational studies. J Natl Cancer Inst 103(1):6176. doi:10.1093/jnci/djq458

24. Rooney AG, McNamara S, Mackinnon M, Fraser M, Rampling R, Carson A, Grant R (2013) Screening for major depressive disorder in adults with cerebral glioma: an initial validation of 3 self-report instruments. Neuro Oncol 15(1):122-129. doi:10.1093/neuonc/nos282

25. Buysse DJ, Reynolds CF 3rd, Monk TH, Berman SR, Kupfer DJ (1989) The Pittsburgh Sleep Quality Index: a new instrument for psychiatric practice and research. Psychiatry Res 28(2):193-213. doi:10.1016/0165-1781(89)90047-4

26. Johns MW (1991) A new method for measuring daytime sleepiness: the Epworth sleepiness scale. Sleep 14(6):540-545. doi:10.1093/sleep/14.6.540

27. Agasi-Idenburg C, Velthuis M, Wittink H (2010) Quality criteria and user-friendliness in self-reported questionnaires on cancer-related fatigue: a review. J Clin Epidemiol 63(7):705-711. doi:10.1016/j.jclinepi.2009.08.027

28. Smets EM, Garssen B, Bonke B, De Haes JC (1995) The Multidimensional Fatigue Inventory (MFI) psychometric qualities of an instrument to assess fatigue. J Psychosom Res 39(3):315-325. doi:10.1016/0022-3999(94)00125-o

29. Asher A, Fu JB, Bailey C, Hughes JK (2016) Fatigue among patients with brain tumors. CNS Oncol 5(2):91-100. doi:10.2217/cns-2015-0008

30. Tartavoulle TM, Karpinski AC, Aubin A, Kluger BM, Distler O, Saketkoo LA (2018) Multidimensional fatigue in pulmonary hypertension: prevalence, severity and predictors. ERJ Open Res 4 (1). doi:10.1183/23120541.00079-2017

31. Tian J, Hong JS (2012) Validation of the Chinese version of Multidimensional Fatigue Inventory-20 in Chinese patients with cancer. Support Care Cancer 20(10):2379-2383. doi:10.1007/s00520-011-1357-8

32. Bunevicius A (2017) Reliability and validity of the SF-36 Health Survey Questionnaire in patients with brain tumors: a cross-sectional study. Health Qual Life Outcomes 15(1):92. doi:10.1186/s12955-017-0665-1

33. Li L, Wang HM, Shen Y (2003) Chinese SF-36 Health Survey: translation, cultural adaptation, validation, and normalisation. J Epidemiol Community Health 57(4):259-263. doi:10.1136/jech.57.4.259

34. Aprile I, Chiesa S, Padua L, Di Blasi C, Arezzo MF, Valentini V, Di Stasio E, Balducci M (2015) Occurrence and predictors of the fatigue in high-grade glioma patients. Neurol Sci 36(8):1363-1369. doi:10.1007/s10072-015-2111-7

35. Schei S, Solheim O, Jakola AS, Sagberg LM (2020) Perioperative fatigue in patients with diffuse glioma. J Neurooncol 147(1):97-107. doi:10.1007/s11060-020-03403-0

36. van Coevorden-van Loon EMP, Coomans MB, Heijenbrok-Kal MH, Ribbers GM, van den Bent MJ (2017) Fatigue in patients with low grade glioma: systematic evaluation of assessment and prevalence. J Neurooncol 133(2):237-246. doi:10.1007/s11060-017-2454-4

37. Nassiri F, Suppiah S, Wang JZ, Badhiwala JH, Juraschka K, Meng Y, Nejad R, Au K, Willmarth NE, Cusimano M, Zadeh G (2020) How to live with a meningioma: experiences, symptoms, and challenges reported by patients. Neurooncol Adv 2(1):vdaa086. doi:10.1093/noajnl/vdaa086

38. Langegard U, Johansson B, Bjork-Eriksson T, Fransson P, Ohlsson-Nevo E, Sjovall K, Ahlberg K (2019) Symptom Clusters in Patients With Brain Tumors Undergoing Proton Beam Therapy. Oncol Nurs Forum 46(3):349-363. doi:10.1188/19.0NF.349-363

39. Horne CE, Johnson S, Crane PB (2019) Comparing comorbidity measures and fatigue post myocardial infarction. Appl Nurs Res 45:1-5. doi:10.1016/j.apnr.2018.11.004

40. Benjamin EJ, Virani SS, Callaway CW, Chamberlain AM, Chang AR, Cheng S, Chiuve SE, Cushman M, Delling FN, Deo R, de Ferranti SD, Ferguson JF, Fornage M, Gillespie C, Isasi CR, Jimenez MC, Jordan LC, Judd SE, Lackland D, Lichtman JH, Lisabeth L, Liu S, Longenecker CT, Lutsey PL, Mackey JS, Matchar DB, Matsushita K, Mussolino ME, Nasir K, O'Flaherty M, Palaniappan LP, Pandey A, Pandey DK, Reeves MJ, Ritchey MD, Rodriguez CJ, Roth GA, Rosamond WD, Sampson UKA, Satou GM, Shah SH, Spartano NL, Tirschwell DL, Tsao CW, Voeks JH, Willey JZ, Wilkins JT, Wu JH, Alger HM, Wong SS, Muntner P, American Heart Association Council on E, Prevention Statistics Stroke Statistics C S (2018) Heart Disease and Stroke Statistics-2018 Update: A Report From the American Heart Association. Circulation 137(12):e67-e492. doi:10.1161/CIR.0000000000000558

41. Kim SR, Shin YS, Kim JH, Choi M, Yoo SH (2017) Differences in Type Composition of Symptom Clusters as Predictors of Quality of Life in Patients with Meningioma and Glioma. World Neurosurg 98:50-59. doi:10.1016/j.wneu.2016.10.085

42. Moradi A, Semnani V, Djam H, Tajodini A, Zali AR, Ghaemi K, Nikzad N, Madani-Civi M (2008) Pathodiagnostic parameters for meningioma grading. J Clin Neurosci 15(12):1370-1375. doi:10.1016/j.jocn.2007.12.005

43. Spierings EL, van Hoof MJ (1997) Fatigue and sleep in chronic headache sufferers: an age- and sex-controlled questionnaire study. Headache 37(9):549552. doi:10.1046/j.1526-4610.1997.3709549.x 
44. Sadighi ZS, Ness KK, Hudson MM, Morris EB, Ledet DS, Pui CH, Howard SC, Krull KR, Browne E, Crom D, Hinds PS, Zhu L, Srivastava D, Khan RB (2014) Headache types, related morbidity, and quality of life in survivors of childhood acute lymphoblastic leukemia: a prospective cross sectional study. Eur $\mathrm{J}$ Paediatr Neurol 18(6):722-729. doi:10.1016/j.ejpn.2014.06.006

45. Goebel S, Mehdorn HM (2018) A missing piece? Neuropsychiatric functioning in untreated patients with tumors within the cerebellopontine angle. J Neurooncol 140(1):145-153. doi:10.1007/s11060-018-2944-z

46. Bunevicius A, Tamasauskas S, Deltuva V, Tamasauskas A, Radziunas A, Bunevicius R (2014) Predictors of health-related quality of life in neurosurgical brain tumor patients: focus on patient-centered perspective. Acta Neurochir (Wien) 156(2):367-374. doi:10.1007/s00701-013-1930-7

47. Armstrong TS, Shade MY, Breton G, Gilbert MR, Mahajan A, Scheurer ME, Vera E, Berger AM (2017) Sleep-wake disturbance in patients with brain tumors. Neuro Oncol 19(3):323-335. doi:10.1093/neuonc/now119

48. Jeon MS, Dhillon HM, Agar MR (2017) Sleep disturbance of adults with a brain tumor and their family caregivers: a systematic review. Neuro Oncol 19(8):1035-1046. doi:10.1093/neuonc/nox019

49. Brown PD, Ballman KV, Rummans TA, Maurer MJ, Sloan JA, Boeve BF, Gupta L, Tang-Wai DF, Arusell RM, Clark MM, Buckner JC (2006) Prospective study of quality of life in adults with newly diagnosed high-grade gliomas. J Neurooncol 76(3):283-291. doi:10.1007/s11060-005-7020-9

50. Hossain JL, Ahmad P, Reinish LW, Kayumov L, Hossain NK, Shapiro CM (2005) Subjective fatigue and subjective sleepiness: two independent consequences of sleep disorders? J Sleep Res 14(3):245-253. doi:10.1111/j.1365-2869.2005.00466.x

51. Gielissen MF, Verhagen CA, Bleijenberg G (2007) Cognitive behaviour therapy for fatigued cancer survivors: long-term follow-up. Br J Cancer 97(5):612618. doi:10.1038/sj.bjc.6603899

52. Mitchell SA, Hoffman AJ, Clark JC, DeGennaro RM, Poirier P, Robinson CB, Weisbrod BL (2014) Putting evidence into practice: an update of evidencebased interventions for cancer-related fatigue during and following treatment. Clin J Oncol Nurs 18 Suppl:38-58. doi:10.1188/14.CJON.S3.38-58

53. Tian L, Lu HJ, Lin L, Hu Y (2016) Effects of aerobic exercise on cancer-related fatigue: a meta-analysis of randomized controlled trials. Support Care Cancer 24(2):969-983. doi:10.1007/s00520-015-2953-9

\section{Tables}

Table 1 Correlations between demographic and fatigue in meningioma patients $(\mathrm{N}=120)$ 


\begin{tabular}{|c|c|c|c|c|c|c|c|c|c|c|c|c|c|}
\hline \multirow[t]{2}{*}{ Variables } & \multirow[t]{2}{*}{ Description } & \multicolumn{2}{|c|}{ Total Fatigue } & \multicolumn{2}{|l|}{$\begin{array}{l}\text { General } \\
\text { Fatigue }\end{array}$} & \multicolumn{2}{|c|}{$\begin{array}{l}\text { Physical } \\
\text { Fatigue }\end{array}$} & \multicolumn{2}{|c|}{$\begin{array}{l}\text { Reduced } \\
\text { Activity }\end{array}$} & \multicolumn{2}{|c|}{$\begin{array}{l}\text { Reduced } \\
\text { Motivation }\end{array}$} & \multicolumn{2}{|c|}{ Mental Fatigue } \\
\hline & & $r$ & $P$ & $r$ & $P$ & $r$ & $P$ & $r$ & $P$ & $r$ & $P$ & $r$ & $P$ \\
\hline Gender, female $^{a}$ & $88(73.3)$ & 0.077 & 0.404 & 0.257 & 0.005 & 0.031 & 0.736 & -0.053 & 0.563 & 0.017 & 0.853 & -0.016 & 0.862 \\
\hline Age, years ${ }^{b}$ & $\begin{array}{l}59.29 \pm \\
11.38\end{array}$ & 0.175 & 0.056 & -0.015 & 0.874 & 0.155 & 0.092 & 0.226 & 0.013 & 0.320 & $<0.001$ & -0.070 & 0.450 \\
\hline $\mathrm{BMI}, \mathrm{kg} / \mathrm{m}^{2} \mathrm{~b}$ & $\begin{array}{l}24.17 \pm \\
3.61\end{array}$ & -0.162 & 0.078 & -0.171 & 0.062 & -0.137 & 0.135 & -0.104 & 0.260 & -0.172 & 0.060 & -0.102 & 0.268 \\
\hline Place of residence ${ }^{a}$ & & 0.002 & 0.987 & -0.028 & 0.765 & 0.004 & 0.963 & -0.017 & 0.855 & -0.013 & 0.888 & -0.002 & 0.983 \\
\hline Urban & 38 (31.7) & & & & & & & & & & & & \\
\hline Rural & $82(68.3)$ & & & & & & & & & & & & \\
\hline Marital status ${ }^{a}$ & & 0.102 & 0.268 & -0.002 & 0.985 & 0.141 & 0.124 & 0.125 & 0.172 & 0.086 & 0.349 & 0.073 & 0.428 \\
\hline Married & 110 (91.7) & & & & & & & & & & & & \\
\hline Other & $10(8.3)$ & & & & & & & & & & & & \\
\hline Educational level a & & -0.024 & 0.795 & 0.014 & 0.882 & -0.043 & 0.642 & 0.033 & 0.717 & -0.042 & 0.651 & 0.135 & 0.142 \\
\hline$\leq 9$ years & $100(83.3)$ & & & & & & & & & & & & \\
\hline$>9$ years & $20(16.7)$ & & & & & & & & & & & & \\
\hline Employment, yes a & $74(61.7)$ & -0.182 & 0.047 & -0.031 & 0.734 & -0.183 & 0.045 & -0.183 & 0.046 & -0.235 & 0.010 & -0.012 & 0.898 \\
\hline $\begin{array}{l}\text { Yearly per capita } \\
\text { income, yuan a a }\end{array}$ & & -0.301 & 0.001 & -0.025 & 0.787 & -0.285 & 0.002 & -0.285 & $<0.001$ & -0.415 & $<0.001$ & -0.067 & 0.466 \\
\hline$<15000$ & 47 (39.2) & & & & & & & & & & & & \\
\hline $15000-33000$ & $34(28.3)$ & & & & & & & & & & & & \\
\hline$>33000$ & $39(32.5)$ & & & & & & & & & & & & \\
\hline Tobacco use, yes ${ }^{a}$ & $24(20)$ & -0.064 & 0.488 & -0.276 & 0.002 & -0.032 & 0.729 & 0.064 & 0.487 & -0.001 & 0.995 & 0.007 & 0.936 \\
\hline Alcohol use, yes ${ }^{a}$ & $30(25)$ & -0.085 & 0.357 & -0.153 & 0.096 & -0.079 & 0.391 & 0.038 & 0.684 & -0.015 & 0.870 & -0.029 & 0.755 \\
\hline Physical exercise, yes ${ }^{a}$ & $25(23.8)$ & 0.024 & 0.811 & -0.104 & 0.290 & -0.006 & 0.951 & 0.099 & 0.314 & 0.120 & 0.224 & -0.063 & 0.523 \\
\hline $\begin{array}{l}\text { Menopause status, yes } \\
\text { a }\end{array}$ & $68(80)$ & 0.323 & 0.003 & 0.177 & 0.105 & -0.242 & 0.025 & 0.243 & 0.025 & 0.366 & 0.001 & 0.099 & 0.365 \\
\hline
\end{tabular}

$B M /$ body mass index

a Values are presented as the number (\%)

${ }^{b}$ Values are presented as the mean $\pm S D$

Italicized values are those considered statistically significant

Table 2 Correlations between clinical, psychological characteristics and fatigue in meningioma patients $(\mathrm{N}=120)$ 


\begin{tabular}{|c|c|c|c|c|c|c|c|c|c|c|c|c|c|}
\hline \multirow[t]{2}{*}{ Variables } & \multirow[t]{2}{*}{ Description } & \multicolumn{2}{|c|}{ Total Fatigue } & \multicolumn{2}{|c|}{ General Fatigue } & \multicolumn{2}{|c|}{$\begin{array}{l}\text { Physical } \\
\text { Fatigue }\end{array}$} & \multicolumn{2}{|c|}{$\begin{array}{l}\text { Reduced } \\
\text { Activity }\end{array}$} & \multicolumn{2}{|c|}{$\begin{array}{l}\text { Reduced } \\
\text { Motivation }\end{array}$} & \multicolumn{2}{|c|}{ Mental Fat } \\
\hline & & $r$ & $P$ & $r$ & $P$ & $r$ & $P$ & $r$ & $P$ & $r$ & $P$ & $r$ & $F$ \\
\hline $\begin{array}{l}\text { Time since diagnosis, } \\
\text { months }{ }^{c} \text { months }\end{array}$ & $\begin{array}{l}0.33(0.23 \\
1)\end{array}$ & 0.109 & 0.238 & 0.061 & 0.509 & 0.124 & 0.176 & 0.150 & 0.101 & -0.020 & 0.825 & 0.202 & 0 \\
\hline Comorbidity, yes a & $67(55.8)$ & 0.186 & 0.042 & 0.180 & 0.049 & 0.175 & 0.056 & 0.174 & 0.057 & 0.119 & 0.194 & 0.034 & 0 \\
\hline Hospitalization, days ${ }^{c}$ & $1(0,1)$ & 0.074 & 0.420 & 0.139 & 0.130 & 0.031 & 0.734 & 0.036 & 0.699 & 0.016 & 0.858 & 0.129 & 0 \\
\hline $\begin{array}{l}\text { Initial presenting symptoms } \\
\text { a }\end{array}$ & & -0.068 & 0.460 & -0.150 & 0.103 & 0.002 & 0.986 & 0.001 & 0.996 & -0.120 & 0.192 & 0.001 & 0 \\
\hline Intracranial hypertension & $76(63.3)$ & & & & & & & & & & & & \\
\hline Epilepsy & $7(5.8)$ & & & & & & & & & & & & \\
\hline Neurological deficits & $27(22.5)$ & & & & & & & & & & & & \\
\hline Others & $10(8.3)$ & & & & & & & & & & & & \\
\hline Maximal diameter, $\mathrm{cm}^{\mathrm{c}}$ & $3.6(2.9,5)$ & 0.288 & 0.002 & 0.144 & 0.119 & 0.288 & 0.001 & 0.263 & 0.004 & 0.280 & 0.002 & 0.177 & 0 \\
\hline Tumor lateralization a & & 0.063 & 0.493 & 0.097 & 0.293 & 0.098 & 0.288 & 0.030 & 0.744 & 0.042 & 0.648 & -0.082 & 0 \\
\hline Left & $53(44.2)$ & & & & & & & & & & & & \\
\hline Right & $57(47.5)$ & & & & & & & & & & & & \\
\hline Bilateral & $10(8.3)$ & & & & & & & & & & & & \\
\hline Tumor location ${ }^{a}$ & & 0.095 & 0.304 & -0.019 & 0.838 & 0.023 & 0.800 & 0.079 & 0.393 & 0.197 & 0.031 & 0.121 & 0 \\
\hline Skull base & 40 (33.3) & & & & & & & & & & & & \\
\hline Non-skull base & $80(66.7)$ & & & & & & & & & & & & \\
\hline WHO grade ${ }^{a}$ & & 0.198 & 0.032 & 0.159 & 0.088 & 0.196 & 0.034 & 0.142 & 0.128 & 0.175 & 0.059 & 0.130 & 0 \\
\hline Grade I & $86(73.5)$ & & & & & & & & & & & & \\
\hline Grade II & $28(23.9)$ & & & & & & & & & & & & \\
\hline 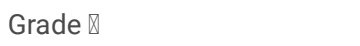 & $3(2.6)$ & & & & & & & & & & & & \\
\hline Hemoglobin, $g / L^{c}$ & $\begin{array}{l}128(122 \\
136)\end{array}$ & -0.005 & 0.958 & -0.145 & 0.114 & -0.029 & 0.750 & 0.027 & 0.769 & 0.006 & 0.944 & 0.021 & 0 \\
\hline Erythrocyte count, $10^{12} / \mathrm{L}^{b}$ & $4.26 \pm 0.43$ & -0.057 & 0.539 & -0.155 & 0.090 & -0.033 & 0.721 & -0.036 & 0.694 & -0.066 & 0.476 & -0.016 & 0 \\
\hline Serum albumin, $g / L^{c}$ & $\begin{array}{l}38.5 \\
(36.83 \\
40.80)\end{array}$ & -0.074 & 0.425 & 0.004 & 0.962 & -0.058 & 0.527 & -0.086 & 0.348 & -0.128 & 0.163 & -0.137 & 0 \\
\hline $\begin{array}{l}\text { Serum } \\
\text { potassium, mmol// }{ }^{c}\end{array}$ & $\begin{array}{l}3.7(3.5 \\
4.0)\end{array}$ & -0.109 & 0.236 & -0.141 & 0.124 & -0.054 & 0.556 & -0.078 & 0.399 & -0.100 & 0.278 & -0.049 & 0 \\
\hline Serum sodium, $\mathrm{mmol} / \mathrm{L}^{\mathrm{c}}$ & $\begin{array}{l}141(140 \\
142)\end{array}$ & 0.205 & 0.025 & 0.106 & 0.247 & 0.119 & 0.195 & 0.195 & 0.033 & 0.331 & $<0.001$ & 0.053 & 0 \\
\hline BI score ${ }^{c}$ & $\begin{array}{l}100(100 \\
100)\end{array}$ & -0.380 & $<0.001$ & -0.216 & $<0.001$ & -0.381 & $<0.001$ & -0.380 & $<0.001$ & -0.321 & $<0.001$ & -0.374 & $<$ \\
\hline KPS score ${ }^{c}$ & $90(90,90)$ & -0.503 & $<0.001$ & -0.336 & $<0.001$ & -0.454 & $<0.001$ & -0.516 & $<0.001$ & -0.463 & $<0.001$ & -0.426 & $<$ \\
\hline Headache, yes ${ }^{a}$ & $25(23.8)$ & 0.194 & 0.034 & 0.252 & 0.005 & 0.177 & 0.053 & 0.094 & 0.310 & 0.157 & 0.087 & 0.131 & 0 \\
\hline MMSE score ${ }^{\mathrm{c}}$ & $26(23,29)$ & -0.314 & 0.001 & -0.202 & 0.029 & -0.307 & 0.001 & -0.296 & 0.001 & -0.278 & 0.002 & -0.142 & 0 \\
\hline Anxiety, yes ${ }^{a}$ & $23(19.2)$ & 0.562 & $<0.001$ & 0.487 & $<0.001$ & 0.559 & $<0.001$ & 0.480 & $<0.001$ & 0.446 & $<0.001$ & 0.512 & $<$ \\
\hline Depression, yes ${ }^{a}$ & $26(21.7)$ & 0.607 & $<0.001$ & 0.517 & $<0.001$ & 0.593 & $<0.001$ & 0.555 & $<0.001$ & 0.499 & $<0.001$ & 0.453 & $<$ \\
\hline Sleep disturbances, yes ${ }^{\text {a }}$ & $52(43.3)$ & 0.412 & $<0.001$ & 0.354 & $<0.001$ & 0.359 & $<0.001$ & 0.299 & 0.001 & 0.339 & $<0.001$ & 0.236 & 0 \\
\hline ESS score ${ }^{c}$ & $3(1,6)$ & 0.239 & 0.011 & 0.113 & 0.232 & 0.192 & 0.041 & 0.212 & 0.023 & 0.293 & 0.002 & 0.239 & 0 \\
\hline
\end{tabular}

WHO world health organization, B/Barthel index, KPS Karnofsky performance score, MMSE Mini-mental State Examination, ESS Epworth Sleepiness Scale 
a Values are presented as the number (\%)

${ }^{b}$ Values are presented as the mean $\pm S D$

${ }^{\mathrm{c}}$ Values are presented as the median $\left(25^{\text {th }}\right.$ and $75^{\text {th }}$ percentiles $)$

Italicized values are those considered statistically significant

Table 3 Prevalence and severity of fatigue in meningioma patients $(\mathrm{N}=120)$, measured with the MFI-20 questionnaire

\begin{tabular}{|ll|}
\hline Variables & Description \\
\hline General Fatigue Score $^{c}$ & $10(8,13.75)$ \\
$>12, \mathrm{~N}(\%)$ & $40(33.3 \%)$ \\
\hline Physical Fatigue Score $^{c}$ & $6(4,13)$ \\
$>12, \mathrm{~N}(\%)$ & $33(27.5 \%)$ \\
\hline Reduced Activity Score $^{c}$ & $9(8,14)$ \\
$>12, \mathrm{~N}(\%)$ & $34(28.3 \%)$ \\
\hline Reduced Motivation Score & $8(7,10)$ \\
$>12, \mathrm{~N}(\%)$ & $15(12.5 \%)$ \\
\hline Mental Fatigue Score ${ }^{c}$ & $8(8,10)$ \\
$>12, \mathrm{~N}(\%)$ & $14(11.7 \%)$ \\
\hline Total Fatigue Score ${ }^{c}$ & $43(36,57.75)$ \\
$>60, \mathrm{~N}(\%)$ & $28(23.3 \%)$ \\
\hline
\end{tabular}

${ }^{\mathrm{c}}$ Values are presented as the median $\left(25^{\text {th }}\right.$ and $75^{\text {th }}$ percentiles $)$

Table 4 Linear regression model of meningioma patients with fatigue

\begin{tabular}{|lllllll|}
\hline Predictors & Predictors & $\beta$ & SE & $t$ & $P$ & ष95\% CIV \\
\hline Total Fatigue & Comorbidity, yes & 5.994 & 2.373 & 2.526 & 0.014 & $(1.251,10.737)$ \\
\hline & KPS score & -0.442 & 0.200 & -2.207 & 0.031 & $(-0.841,-0.042)$ \\
\hline & Headache, yes & 5.807 & 2.336 & 2.485 & 0.016 & $(1.136,10.477)$ \\
& Depression, yes & 17.090 & 4.974 & 3.436 & 0.001 & $(7.147,27.033)$ \\
\hline General Fatigue & Sleep disturbances, yes & 4.855 & 2.319 & 2.094 & 0.040 & $(0.219,9.490)$ \\
\hline & ESS score & 1.091 & 0.351 & 3.113 & 0.003 & $(0.390,1.792)$ \\
\hline Physical Fatigue & Aeadache, yes & 1.402 & 0.521 & 2.691 & 0.008 & $(0.369,2.435)$ \\
\hline Reduced Activity & Depression, yes & 2.703 & 1.162 & 2.327 & 0.022 & $(0.400,5.007)$ \\
\hline & KPS score & -0.179 & 0.070 & -2.578 & 0.012 & $(-0.318,-0.040)$ \\
\hline Reduced Motivation & Depression, yes & 4.368 & 1.592 & 2.744 & 0.008 & $(1.189,7.546)$ \\
\hline & Depression, yes & 4.395 & 1.433 & 3.067 & 0.003 & $(1.5333,7.257)$ \\
\hline Mental Fatigue & ESS score & 0.368 & 0.107 & 3.444 & 0.001 & $(0.155,0.582)$ \\
\hline & KPS score & -0.082 & 0.029 & -2.797 & 0.006 & $(-0.141,-0.024)$ \\
\hline & Anxiety, yes & 1.962 & 0.742 & 2.646 & 0.009 & $(0.492,3.433)$ \\
\hline
\end{tabular}

KPS Karnofsky performance score, ESS Epworth Sleepiness Scale

Table 5 Correlations between fatigue status and quality of life in meningiomas patients 


\begin{tabular}{|c|c|c|c|c|c|c|c|}
\hline Variables & Description & Total Fatigue & General Fatigue & Physical Fatigue & Reduced Activity & Reduced Motivation & Mental Fatigue \\
\hline \multicolumn{8}{|l|}{ SF-36 } \\
\hline $\mathrm{PCS}^{\mathrm{C}}$ & $78.63(49.56,92.13)$ & $-0.763^{\star *}$ & $-0.554^{\star \star}$ & $-0.737^{\star \star}$ & $-0.644^{\star \star}$ & $-0.557^{\star *}$ & $-0.417^{\star \star}$ \\
\hline $\operatorname{MCS}^{\mathrm{c}}$ & $86(58.78,92.72)$ & $-0.712^{\star \star}$ & $-0.600^{\star *}$ & $-0.665^{\star *}$ & $-0.588^{\star *}$ & $-0.546^{\star *}$ & $-0.402^{\star \star}$ \\
\hline $\mathrm{PF}^{\mathrm{c}}$ & $95(75,100)$ & $-0.632^{\star *}$ & $-0.442^{\star *}$ & $-0.652^{\star *}$ & $-0.572^{\star \star}$ & $-0.466^{\star *}$ & $-0.462^{\star \star}$ \\
\hline $\mathrm{RP}^{\mathrm{c}}$ & $75(0,100)$ & $-0.688^{\star \star}$ & $-0.464^{\star *}$ & $-0.674^{\star *}$ & $-0.625^{\star *}$ & $-0.517^{\star \star}$ & $-0.326^{\star \star}$ \\
\hline $\mathrm{BP}^{\mathrm{c}}$ & $100(62,100)$ & $-0.346^{\star \star}$ & $-0.242^{\star}$ & $-0.344^{\star \star}$ & $-0.298^{\star}$ & $-0.274^{*}$ & $-0.252^{*}$ \\
\hline $\mathrm{GH}^{\mathrm{c}}$ & $67(45,87)$ & $-0.710^{* *}$ & $-0.576^{\star *}$ & $-0.680^{\star *}$ & $-0.571^{\star *}$ & $-0.499^{* *}$ & $-0.361^{* *}$ \\
\hline $\mathrm{VT}^{\mathrm{c}}$ & $75(55,85)$ & $-0.768^{\star \star}$ & $-0.710^{\star *}$ & $-0.717^{\star \star}$ & $-0.581^{\star \star}$ & $-0.593^{\star *}$ & $-0.463^{\star \star}$ \\
\hline $\mathrm{SF}^{\mathrm{c}}$ & $100(75,100)$ & $-0.601^{\star \star}$ & $-0.365^{\star \star}$ & $-0.630^{\star \star}$ & $-0.583^{\star *}$ & $-0.421^{\star \star}$ & $-0.432^{\star \star}$ \\
\hline $\mathrm{RE}^{\mathrm{c}}$ & $100(0,100)$ & $-0.492^{\star *}$ & $-0.423^{\star \star}$ & $-0.470^{\star *}$ & $-0.441^{\star *}$ & $-0.357^{\star *}$ & $-0.256^{*}$ \\
\hline $\mathrm{MH}^{\mathrm{c}}$ & $76(61,88)$ & $-0.584^{\star \star}$ & $-0.519^{\star \star}$ & $-0.539^{\star \star}$ & $-0.469^{\star \star}$ & $-0.485^{\star \star}$ & $-0.400^{\star \star}$ \\
\hline
\end{tabular}

SF-36 the Short Form 36 Health Survey, PCS physical component scale, MCS mental component scale, $P F$ physical functioning, $R P$ role physical, $B P$ bodily pain, GH general health, $V T$ vitality, $S F$ social functioning, $R E$ role emotional, $M H$ metal health

${ }^{\mathrm{c}}$ Values are presented as the median $\left(25^{\text {th }}\right.$ and $75^{\text {th }}$ percentiles $)$

${ }^{\star \star} P \otimes 0.001,{ }^{*} P<0.01$ 